\title{
La huella ecológica. El establecimiento de indicadores ambientales y su significación para el Derecho*.
}

\section{The ecological footprint. Establishing environmental indicators and their significance for in law}

\author{
ALba Nogueira LóPEZ \\ Catedrática de Derecho Administrativo \\ Universidade de Santiago de Compostela \\ alba.nogueira@usc.es
}

Fecha de recepción: 13 de mayo de 2019 / Fecha de aceptación: 18 de junio de 2019

Resumen: La huella ambiental como posible indicador de comportamiento ambiental ha sido objeto de discusiones en cuanto a su alcance o utilidad en el ámbito científico. Desde un punto de vista jurídico debe examinarse en qué medida es un indicador válido de comportamiento ambiental, sus limitaciones y posibles aplicaciones. También como el Derecho puede incorporar parámetros dotados de una cierta incertidumbre científica para la definición de políticas públicas o la adopción de normas de sostenibilidad.

Resum: La petjada ambiental com a possible indicador de comportament ambiental ha estat objecte de discussions sobre el seu abast o utilitat en l'àmbit científic. Des d'un punt de vista jurídic cal examinar en quina mesura és un indicador vàlid de comportament ambiental, així com les limitacions i possibles aplicacions que té. També cal valorar com el dret pot incorporar paràmetres dotats d'una certa incertesa científica per definir polítiques públiques o adoptar normes de sostenibilitat.

\footnotetext{
* Este trabajo se realiza en el marco del programa "Axudas do programa de consolidación e estruturación de unidades de investigación competitivas. Modalidade grupos con potencial de crecemento" (Xunta de Galicia CN 2012/078)".
} 


\begin{abstract}
The environmental footprint as a possible indicator of environmental behavior has been discussed in terms of its scope or usefulness in the scientific field. From a legal point of view, it must be examined to what extent it is a valid indicator of environmental behavior, its limitations and possible applications. Also, from a legal perspective, if Law can incorporate parameters endowed with a certain scientific uncertainty for the definition of public policies or the adoption of sustainability standards.
\end{abstract}

Palabras clave: huella ambiental - indicadores ambientales - cargas administrativas - aplicaciones jurídicas.

Keywords: environmental footprint - environmental indicators - administrative burdens - legal uses.

Paraules clau: petjada ambiental - indicadors ambientals - càrregues administratives - aplicacions jurídiques.

SUMARIO. 1. La capacidad del planeta tiene límites. La huella ecológica como indicador para la sostenibilidad. 2. El uso de indicadores de comportamiento ambiental 3. Un indicador dúctil que permite medir en distintos planos. 4. La proliferación de indicadores de comportamiento ambiental en el contexto de reducción cargas administrativas. 5. Aplicación de la metodología de la huella ambiental: posibles usos y alcance. 6. Reflexiones conclusivas: El Derecho ante la determinación de los parámetros metodológicos para la medición y el uso de la huella ecológica

\title{
1. LA CAPACIDAD DEL PLANETA TIENE LÍMITES. LA HUELLA ECOLÓGICA COMO INDICADOR PARA LA SOSTENIBILIDAD
}

Vivimos en tiempos de urgencia ambiental. La finitud de los recursos naturales, el deterioro ambiental creciente, el impacto evidente del cambio climático, patrones de consumo insostenibles y la destrucción de los ecosistemas muestran que es necesario caminar hacia un cambio de modelo que haga viable la continuidad del planeta y de las especies que en el vivimos. 
El sexto informe Perspectivas del Medio Ambiente Mundial de la Organización de Naciones Unidas, "Planeta sano, personas sanas", que pretende ser una fuente de información ambiental sólida y basada en pruebas, a fin de "ayudar a los responsables de formular políticas y a toda la sociedad a lograr la dimensión ambiental de la Agenda 2030 para el Desarrollo Sostenible y los objetivos ambientales acordados internacionalmente, y aplicar los acuerdos multilaterales sobre el medio ambiente" (GEO 6, 2019, 7) apunta tendencias claramente preocupantes para la sostenibilidad ambiental y la pervivencia del planeta:

"Las emisiones antropógenas siguen alterando la composición de la atmósfera, lo que da lugar a la contaminación del aire, el cambio climático, la reducción del ozono estratosférico y la exposición a sustancias químicas persistentes, bioacumulativas y tóxicas".

"La contaminación del aire es el principal factor ambiental que contribuye a la carga mundial de morbilidad, y ocasiona entre 6 y 7 millones de muertes prematuras".

"La diversidad genética está disminuyendo, lo que plantea una amenaza para la seguridad alimentaria y la resiliencia de los ecosistemas, incluidos los sistemas agrícolas y la seguridad alimentaria".

"Las poblaciones de especies están disminuyendo y las tasas de extinción de especies están aumentando. En la actualidad, se considera que el $42 \%$ de los invertebrados terrestres, el $34 \%$ de los invertebrados de agua dulce y el $25 \%$ de los invertebrados marinos están en riesgo de extinción."

"La tasa de liberación de gases de efecto invernadero inducida por el hombre está impulsando el aumento del nivel del mar, cambios en la temperatura de los océanos y la acidificación de los océanos. Los arrecifes de coral están siendo devastados por esos cambios".

"Hoy en día la basura marina, compuesta entre otras cosas de plásticos y microplásticos, abunda en todos los océanos, a todas las profundidades".

"La degradación de las tierras y la desertificación han aumentado". "La calidad del agua ha empeorado significativamente desde 1990, debido a la contaminación orgánica y química ocasionada por, entre otros, agentes 
patógenos, fertilizantes, plaguicidas, sedimentos, metales pesados, desechos plásticos y microplásticos, contaminantes orgánicos persistentes y salinidad".

Alcanzar un consenso científico sobre los problemas existentes es una pre-condición para poder abordar los problemas y para diseñar políticas públicas y normas que puedan corregir esa situación y evaluar su cumplimiento. Precisamente en esa función de evaluación o contraste de la eficacia de la acción de mejora ambiental juega un papel central el establecimiento de indicadores que permitan medir de forma fiable, comparable y reproducible la evolución. El Derecho y las políticas públicas necesitan incorporar indicadores elaborados desde el ámbito científico-técnico para orientar y evaluar las soluciones adoptadas.

En ese contexto se desarrolla el concepto de huella ambiental. La definición de WACKERNAGEL y REES $(1997,4)$ decía que la huella ecológica es la medida biofísica directa del capital natural renovable "definido como todos los componentes de la ecosfera y las relaciones estructurales entre ellos, cuya integridad organizativa es esencial para la reproducción continua del sistema mismo". Hay que señalar además que la huella ambiental aparece como una evolución o sofisticación de la huella de carbono. La huella ambiental pretende ir más allá incluyendo un análisis global de impactos con un enfoque de ciclo de vida que abarca, entre otros, el agua y la biodiversidad lo que la hace más exigente, a la par que compleja en cuanto a la medición del nivel de explotación de los recursos (PRAG, 2013, 20).

El proyecto Global Fooprint Network, un think tank que desde 2003 promueve el desarrollo y medición de la huella ecológica, establece que:

"la Huella Ecológica se deriva del seguimiento del área biológicamente productiva que se necesita para satisfacer todas las demandas en competencia de las personas. Estas demandas incluyen espacio para el cultivo de alimentos, la producción de fibra, la regeneración de madera, la absorción de emisiones de dióxido de carbono de la quema de combustibles fósiles y el alojamiento de infraestructura construida. El consumo de un país se calcula sumando las importaciones y restando las exportaciones de su producción nacional.

Todos los productos llevan consigo una cantidad integrada bioproductiva de tierra y mar necesaria para producirlos y secuestrar los desechos asociados. 
Por lo tanto, los flujos de comercio internacional se pueden ver como flujos de Huella.

La Huella Ecológica utiliza los rendimientos de los productos primarios (de tierras de cultivo, bosques, tierras de pastoreo y pesquerías) para calcular el área necesaria para apoyar una actividad determinada.

La biocapacidad se mide calculando la cantidad de tierra y área de mar biológicamente productivas disponibles para proporcionar los recursos que consume una población y absorber sus desechos, dada la tecnología actual y las prácticas de manejo. Para hacer que la biocapacidad sea comparable en el espacio y el tiempo, las áreas se ajustan proporcionalmente a su productividad biológica. Estas áreas ajustadas se expresan en "hectáreas globales". Los países difieren en la productividad de sus ecosistemas, y esto se refleja en las cuentas".

Los datos disponibles de España hablan de una biocapacidad por persona de 1,4 ha. y una huella ecológica por persona de 3,8 ha. por lo que el déficit existente sería de 2,4 ha. (en 1961 era de -1ha., en los años "dorados" pre-crisis había alcanzado las 4,4 ha. en 2007). "Este hecho nos indica que la comunidad se está apropiando de superficies fuera de su territorio, o bien, que está hipotecando y haciendo uso de superficies de las futuras generaciones" (GULLÓN, 2008, 55) en una línea claramente contraria al desarrollo sostenible.

No obstante, existe un debate conceptual intenso sobre la capacidad de este indicador para medir de forma adecuada la complejidad de la sostenibilidad. En un debate doctrinal de ida y vuelta, que explicita con claridad las percepciones diversas sobre el concepto, sus efectos y utilidad, GIAMPIETRO, SALTELLI (2014) y GOLFINGER, WACKERNAGEL, GALLI, LAZARUS, LIN (2014) confrontan dos visiones opuestas sobre su pertinencia y alcance.

GIAMPIETRO, SALTELLI (2014) partiendo de un contundente "huellas ecológicas a la nada" realiza una crítica profunda a la metodología de la huella ecológica de la Global Footprint Network que deriva de los trabajos académicos de REES y WACKERNAGEL. En síntesis, apuntan la debilidad de intentar reconducir a una simple cifra la complejidad de una cuestión multidimensional como la de la sostenibilidad a escala local y global. E inciden en que el éxito mediático del 
concepto, que achacan a su simplicidad, oculta una infravaloración de los impactos medidos por la huella ecológica. Así, "la huella ecológica proporciona estimaciones confortablemente bajas de los niveles de sobreexplotación de los recursos naturales" que no podría trasladarse a ese ritmo de crecimiento para una población global de 9/10 mil millones de personas. Para concluir que "cualquier análisis ecológico serio sobre la sostenibilidad de los actuales patrones implicaría el abandono del cuento de hadas del crecimiento perpetuo a través del progreso técnico (más eficiencia y globalización (más mercado)" (2014, 620).

Por el contrario, en una respuesta directa defendiendo su validez, GOLFINGER, WACKERNAGEL, GALLI, LAZARUS, LIN (2014), mantienen que es una herramienta útil que nunca pretendió ser un instrumento para "manejar la complejidad de la sostenibilidad". No es una así una medida completa de la sostenibilidad, pero ofrece información relevante para un aspecto crítico de la sostenibilidad que es saber el ritmo al que la economía requiere recursos renovables y la capacidad del planeta para regenerarlos. Aunque no abarca todos los datos que en un "mundo ideal" representarían la capacidad regenerativa, si contempla los datos accesibles, consistentes y comparables existentes. También discuten los argumentos de que la huella ecológica es un indicador cambiante o inestable y "media-friendly", indicando que intenta buscar un equilibrio entre la estabilidad y la mejora metodológica y ha permitido generar un debate con base científica sobre los límites ecológicos.

Un intento de sintetizar las debilidades de la huella ambiental (ECOTEC, 2001, 27) además de apuntar el exceso de simplificación de un indicador único y problemas de transparencia, señala que sólo cubre cierto tipo de emisiones $\left(\mathrm{CO}_{2}\right)$; ignora el elemento de escasez de ciertos tipos de tierra; no discrimina el tipo de degradación que produce cada tipo de uso urbano; la unidad de medida (hectárea) es hipotética y podría ser confundida con la unidad geográfica; no distingue entre usos sostenibles e insostenibles del suelo; el método de cálculo no permite al terreno proporcionar servicios de biodiversidad, madera y secuestro de carbono e; ignora el papel de los océanos en la captura de carbono.

Se percibe, en todo caso, en ese debate, y eso es lo que jurídicamente puede ser más interesante analizar, que estamos ante una metodología para cuantificar el comportamiento ambiental y que sus puntos débiles son los habituales para este tipo de indicadores: fiabilidad, comparabilidad, complejidad. 
Estas debilidades, precisamente, son las que dice pretender contrarrestar la Unión Europea publicando una Recomendación de la Comisión de 9 de abril de 2013 sobre el uso de métodos comunes para medir y comunicar el comportamiento ambiental de los productos y las organizaciones a lo largo de su ciclo de vida. En ésta se indica que "es esencial disponer de mediciones e informaciones fiables y correctas sobre el comportamiento ambiental" y "la proliferación actual de métodos e iniciativas diferentes para evaluar y comunicar el comportamiento ambiental está generando confusión y desconfianza". Además de otras cuestiones que analizaremos infra llama la atención que este extenso documento europeo renuncia a dotarse de una definición consistente de huella ecológica. En el apartado de definiciones (2.c) se establece como huella ambiental de los productos [el] "resultado de un estudio de la huella ambiental de los productos basado en el método de la huella ambiental de los productos". Una definición hueca, tautológica y en bucle que no permite discernir el alcance del concepto que la UE usa para construir una metodología de medición del comportamiento ambiental. Aunque en un glosario final si que es más clara, cuando menos porque realiza una definición matizada que se aparta explícitamente de la concepción de sus primeros impulsores en algunos aspectos: "Se refiere a la superficie de tierra productiva y ecosistemas acuáticos necesarios para producir los recursos que consume la población y asimilar los residuos que produce la población, independientemente de dónde estén localizadas la tierra y el agua (Wackernagel y Rees, 1996). La huella ambiental de acuerdo con la presente Guía de la HAO no es igual a la huella ecológica de Wackernagel y Rees: las principales diferencias se señalan en el anexo X de la Guía de HAP (EC-JRC-IES, 2012)".

¿Debe ser este debate conceptual y metodológico aún en marcha un obstáculo para la adopción por el Derecho del concepto de huella ecológica? La incertidumbre científica sobre los contornos y pertinencia de la huella ecológica como indicador ambiental ¿menoscaba su virtualidad jurídica?. ESTEVE $(2009,151-170)$ analizaba la "deriva cientifista del derecho" considerando sorprendente que actualmente el ordenamiento jurídico "ante las reconocidas incertidumbres de la ciencia, se entrega más rendidamente a ella, en vez de desarrollar su potencial de ficción para construir su propio cuadro de certezas". Reivindica, en suma, el modelo de decisión característico del Derecho mediante presunciones ante situaciones de incertidumbre. 
Este trabajo no busca adentrarse por eso en el debate conceptual sobre la huella ecológica sino intentar dibujar cual puede ser su utilidad y/o limitaciones para el Derecho y la formulación y evaluación de políticas ambientales.

\section{EL USO DE INDICADORES DE COMPORTAMIENTO AMBIENTAL}

En la elaboración y evaluación de políticas públicas no es infrecuente el uso de indicadores científicos (valores límite, índices de contaminación), económicos (PIB) o, incluso, sociales (condiciones de vida, morbilidad). Hay también propuestas sugerentes que buscan engarzar los límites planetarios y sociales en un marco conceptual conjunto que permite visualizar y cuantificar los techos ambientales y las carencias sociales buscando un equilibrio en el bienestar de un espacio dado, como la "economía del donus" enunciada por Kate RAWORTH en un trabajo para Oxfam en 2012 y posteriormente desarrollada en otros estudios.

En cambio, la evaluación ambiental de la efectividad jurídica es algo así como "el mito de la búsqueda del Grial" (PRIEUR, 2018, XVI). Es posible que antes de adentrarnos a desentrañar los problemas jurídicos que plantean indicadores extraídos de otros ámbitos del conocimiento, debiéramos desarrollar herramientas jurídicas de medición de la efectividad de las normas "desde la cuna hasta la tumba". PRIEUR (2018) realiza precisamente un interesante esfuerzo de poner en pie un conjunto de indicadores jurídicos que permitan medir el Derecho: la existencia de la regla; la validez de la regla; la entrada en vigor de la regla; la invocabilidad de la regla; la conciencia de la regla; la substancia de la regla; el progreso o la regresión de la regla; la precisión de la regla; el control administrativo de la regla; el control jurisdiccional de la regla; la sanción de la regla; la aplicación de las sanciones. Esta construcción metodológica pretende visibilizar y corregir uno de los problemas más frecuentemente señalados del Derecho ambiental, la distancia entre las normas y su aplicación efectiva.

Este podría ser un primer debate que plantea el uso por el Derecho de indicadores cuantitativos. ¿Usamos metodologías cuantitativas para medir el Derecho o usamos indicadores cuantitativos procedentes de la ciencia para construir la norma de conducta jurídica?. En el primer caso cuantificamos Derecho (¿hay norma?, ¿se ha aplicado?, ¿se incumple habitualmente?, ¿cuántas sanciones lo indican?), en el 
segundo cuantificamos para el Derecho (ej. un índice que fija el umbral tolerable de emisión de una substancia).

La huella ambiental formaría parte de este segundo bloque de indicadores cualitativos. Metodologías de medición procedentes del conocimiento científico que nos permiten establecer límites, fijar objetivos, cuantificar logros y evaluar políticas, todo ello en función del uso que se les atribuya al incorporarlos a las normas y/o políticas públicas.

"Es esencial disponer de mediciones e informaciones fiables y correctas sobre el comportamiento ambiental de los productos y de las organizaciones para la toma de decisiones ambientales a diversos niveles", afirma el primer considerando de la Recomendación de la UE de 2013 sobre la huella ecológica. Esta finalidad es similar a la que buscarían los criterios ecológicos de la ecoetiqueta de la UE. El Reglamento 6/2010, de 25 de noviembre de 2009, relativo a la etiqueta ecológica de la UE pretende también que "los criterios que deben cumplir los productos para llevar la etiqueta ecológica de la UE se basen en el mejor comportamiento ambiental alcanzado por los productos en el mercado comunitario. Esos criterios deben ser fáciles de entender y utilizar y deben basarse en pruebas científicas tomando en consideración los últimos avances tecnológicos"(considerando 5). Unas pretensiones en gran medida coincidentes con las de la metodología para la huella ambiental. La disponibilidad de datos, "la pertinencia, la aceptación, la credibilidad, la facilidad de uso y la solidez" aparecen también como criterios para la fijación de indicadores para la economía circular en su Marco de seguimiento (COM (2018) 29 final, 6). En definitiva, el establecimiento de indicadores de comportamiento ambiental no ha sido ajeno al Derecho ambiental de la UE aunque se percibe la dificultad de alcanzar criterios con solidez científica que permitan su comparabilidad, fiabilidad y una cierta simplicidad en la obtención de datos y uso.

La Unión Europea en diversos documentos de la década de los noventa del pasado siglo y en el Sexto programa de acción en materia de medio ambiente había apostado por reforzar la elaboración de indicadores de comportamiento ambiental en una línea de diversificación contraria a la condensación en un indicador único. La Comunicación (COM 94670 final) Directrices que debe seguir la UE en relación con los indicadores ambientales y la contabilidad ecológica nacional quería impulsar un sistema europeo de indicadores ambientales. Igualmente el Sexto programa de 
acción (COM/2001/0031 final) optaba por reforzar la evaluación de las políticas ambientales con un "conjunto amplio" de indicadores sectoriales: "La Comisión tiene intención de desarrollar un proceso de evaluación sistemático para mejorar las políticas futuras y su aplicación, y para poder evaluar posibles evoluciones futuras. Para la evaluación intermedia de los progresos obtenidos en la aplicación del presente programa, será crucial poder disponer de un conjunto amplio de indicadores".

La nota distintiva de la huella ecológica con respecto a otros indicadores de comportamiento ambiental es la condensación de la información en un único parámetro: la medida biofísica directa del capital natural renovable. Ya hemos apuntado algunas críticas doctrinales a esa simplicidad, que en suma es una crítica a que la diversidad de factores inherentes a la sostenibilidad pueda ser representada con un único indicador.

Los problemas de validez temporal, geográfica y, fundamentalmente, la limitación en la caracterización de los impactos, aparecen también como puntos débiles en el estudio comparativo sobre metodologías de análisis del ciclo de vida realizado por la Comisión europea en 2010 que reproducimos infra.

Principios generales de la metodología de la huella ambiental

\begin{tabular}{|l|l|}
\hline Principio & Comentario \\
\hline $\begin{array}{l}\text { Propósito pretendido de } \\
\text { la metodología: }\end{array}$ & $\begin{array}{l}\text { Proporcionar un indicador del área biológica } \\
\text { productiva necesaria para satisfacer la demanda } \\
\text { humana. Enfoque compuesto. }\end{array}$ \\
\hline $\begin{array}{l}\text { Punto intermedio/punto } \\
\text { final: }\end{array}$ & $\begin{array}{l}\text { Método de punto final que calcula el área ocupada } \\
\text { para proporcionar los recursos necesarios y } \\
\text { absorber los residuos que genera, usando las } \\
\text { tecnologías predominantes y la gestión de los } \\
\text { recursos. }\end{array}$ \\
\hline
\end{tabular}




\begin{tabular}{|c|c|}
\hline \begin{tabular}{|l|l} 
Manejo de alternativas: & c \\
c \\
á \\
a \\
$\mathrm{b}$ \\
$\mathrm{c}$ \\
$\mathrm{c}$
\end{tabular} & $\begin{array}{l}\text { Sólo áreas productivas biológicas, las zonas } \\
\text { construidas se consideran con el mismo valor que el } \\
\text { área de cultivo primario. Se asume que los océanos } \\
\text { absorben el } 35 \% \text { de las emisiones y el resto por los } \\
\text { bosques. Los residuos nucleares se consideran } \\
\text { convirtiendo el output de la electricidad generada en } \\
\mathrm{CO}_{2} \text {. }\end{array}$ \\
\hline $\begin{array}{l}\text { Incertidumbre sobre los } \\
\text { datos: }\end{array}$ & $\begin{array}{l}\text { Infraestimación de la realidad. No se menciona } \\
\text { ninguna cifra de incertidumbre. }\end{array}$ \\
\hline Validez regional: & $\begin{array}{l}\text { Las diferencias regionales se manejan a nivel } \\
\text { estatal, produciendo factores de rendimiento } \\
\text { específicos. }\end{array}$ \\
\hline Validez temporal: & $\begin{array}{l}\text { Modelo altamente dependiente de avatares } \\
\text { temporales. }\end{array}$ \\
\hline Horizonte temporal: & - \\
\hline $\begin{array}{l}\text { ¿Como se garantiza la } \\
\text { consistencia en ellit } \\
\text { tratamiento de los } \\
\text { diferentes impactos? } \\
\text { - ¿En la caracterización? } \\
\text { - ¿En la normalización y } \\
\text { peso? }\end{array}$ & $\begin{array}{l}\text { Se consideran } 5 \text { tipos de ocupación directa de la } \\
\text { tierra (tierras de cultivo, pastos, bosques, } \\
\text { pesquerías, áreas edificadas) y dos ocupaciones } \\
\text { indirectas de la tierra (combustibles fósiles y energía } \\
\text { nuclear). Los siete factores se expresan en } \\
\text { hectáreas globales (estandarización basada en la } \\
\text { productividad), que se normalizan a las hectáreas } \\
\text { productivas biológicas reales disponibles en la tierra. }\end{array}$ \\
\hline $\begin{array}{l}\text { Impactos de punto medio } \\
\text { cubiertos: }\end{array}$ & No procede \\
\hline $\begin{array}{l}\text { Impactos de punto final } \\
\text { cubiertos: }\end{array}$ & No procede \\
\hline \begin{tabular}{l|l} 
Número aproximado de & 5 \\
substancias cubiertas: & 1
\end{tabular} & 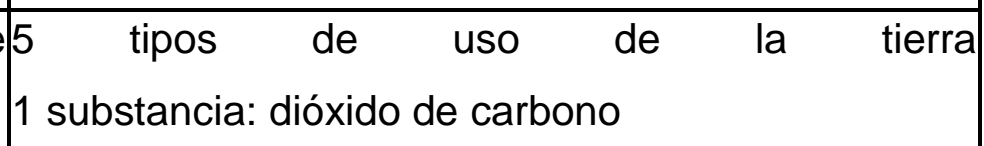 \\
\hline
\end{tabular}




\begin{tabular}{|l|l|}
\hline Otras observaciones: & Ninguna \\
\hline Elaboración: European Commission (2010,60), ILCD Handbook: Analysing of existing
\end{tabular}

Environmental Impact Assessment methodologies for use in Life Cycle Assessment. First edition

Si tomamos como referencia la estrategia de Economía Circular de la UE, que se anuncia como la más ambiciosa actualmente en este momento en términos ambientales, su Marco de seguimiento afirma que "no existe un indicador universalmente reconocido de "circularidad», el número de indicadores fiables y listos para su uso a fin de describir las tendencias más pertinentes es escaso. No sería posible con una única medida o puntuación reflejar adecuadamente la complejidad y las múltiples dimensiones de la transición hacia una economía circular" (COM(2018) 29 final,2). A pesar de esa afirmación sobre la necesidad de contar con una gama de indicadores para evaluar la economía circular, lo cierto es que más recientemente en el Informe sobre la aplicación del Plan de acción para la economía circular (COM(2019) 190 final, 4) la Comisión Europea expresa su valoración positiva del método de la huella ambiental de los productos (HAP) y el método de la huella ambiental de las organizaciones (HAO) ya que "permiten a las empresas declarar especificaciones ecológicas fiables, reproducibles y comparables. Los métodos permiten identificar puntos problemáticos desde un punto de vista medioambiental y apoyar a las empresas en la ecologización de su cadena de suministros y en sus esfuerzos por ser más sostenibles y circulares. Asimismo, los consumidores podrán tomar decisiones con conocimiento de causa y basadas en información fiable". Se pone también en valor que ya existe una fase piloto contrastada en la que participaron unas trescientas empresas de veintisiete sectores distintos y más de 2000 partes interesadas durante cinco años para poner a prueba estos métodos, que "consideran mejores prácticas en la evaluación del ciclo de vida".

Es preciso, por tanto, examinar en qué aspectos puede ser útil la huella ecológica como indicador ambiental y las insuficiencias o carencias que podría presentar.

\section{UN INDICADOR DÚCTIL QUE PERMITE MEDIR EN DISTINTOS PLANOS}


Un valor interesante que puede tener la huella ambiental es su capacidad de reconducir a un indicador comparable la medida biofísica directa del capital natural renovable, que tanto puede ser de aplicación en un plano micro (productos), como en uno macro (sostenibilidad de un determinado territorio).

Diversas autoras (ALONSO IBÁÑEZ, 2016, 197; GALERA RODRIGO, 2016, 490-493) han apuntado como esa coordinación vertical o ese eslabón entre los distintos planos de acción es uno de los fallos de las políticas públicas ambientales en general o de algunas más específicas (lucha contra el cambio climático) y expresado la necesidad de formular metodologías de intervención que puedan poner en relación los distintos niveles.

En línea con esas reflexiones la existencia de indicadores de comportamiento ambiental, como la huella ambiental, que pueden ofrecer un dato comparable desde el nivel de los productos hasta el de organizaciones de tamaños muy diversos (ayuntamientos, países, regiones...) podría actuar como un complemento adecuado a esas políticas públicas engarzadas que se demandan para hacer frente al reto ambiental. La literatura científica ha desarrollado esa metodología para diferentes escalas partiendo de los estudios de WACKERNAGEL y REES para adaptarlos a la escala global/estatal (WWF, 2000), a las ciudades (FOLKE, 1997), a las empresas (CHAMBERS, LEWIS, 2001) y a los individuos y productos (ECOTEC, 2001, 17).

Sin embargo, también hay elementos que condicionan precisamente esa comparabilidad que sería el elemento comunicativamente más fuerte de la huella ambiental. Por un lado, la inexistencia de datos desagregados de suficiente calidad que dificulta recoger los flujos de materiales y energía en el nivel regional/local o empresarial. Por ese motivo, la forma de obtener información para los niveles meso y micro parte de información no oficial y obliga en ciertos casos a realizar simplificaciones o asunciones que pueden restar fiabilidad al resultado. Además, esta diversidad de escalas superpone unas divisiones de naturaleza fundamentalmente política a un hecho ambiental que difícilmente conoce fronteras por lo que el ajuste en las mediciones parece puramente convencional o tentativo más que riguroso. Estas circunstancias conducen a que se entienda que el nivel en el que existe una información más robusta y comparable de los elementos que integran la huella ambiental es el estatal ya que una buena parte de los datos se producen de forma estandarizada a nivel internacional (ECOTEC, 2001, 18). 
Aunque no se han determinado sesgos concretos en las metodologías empleadas, se advierte que los sistemas de huella ambiental o de carbono pueden discriminar a proveedores en función de su distancia a los mercados de referencia por las necesidades de transporte que deberían ser computadas (PRAG, 2013, 47). Por otro lado, esa comparabilidad depende de que la información existente para cuantificar los datos, muy ligada a las condiciones locales, sea transparente. También hay iniciativas internacionales (UNEP, UE, EPA estadounidense) para cooperar en la definición del elemento clave, las reglas de categorías de productos a los que se aplica el análisis de ciclo de vida (PRAG, 2013, 48).

\section{LA PROLIFERACIÓN DE INDICADORES DE COMPORTAMIENTO AMBIENTAL EN EL CONTEXTO DE REDUCCIÓN CARGAS ADMINISTRATIVAS}

La reducción de cargas administrativas se repite como un mantra en todas las iniciativas europeas de los últimos años. Desde que en 2006 la Unión Europa lanzara la iniciativa "Legislar mejor" y pusiera en marcha el programa de adecuación y eficacia de la reglamentación de la Comisión Europea (REFIT), acompañado de indicadores y una plataforma para impulsar una evaluación sistemática de las medidas propuestas, este ha sido un vector principal de acción europea.

En línea con este objetivo existe una cierta cautela hacia el papel que pueden jugar indicadores como la huella ambiental en un incremento de las cargas administrativas. De hecho esta advertencia figura de forma nítida en la Recomendación sobre la huella ecológica (2013/179/UE):

"El objetivo último de la iniciativa consiste en paliar la fragmentación del mercado interior derivada de la disponibilidad de múltiples métodos de medición del comportamiento ambiental. La Comisión considera que, antes de que su aplicación sea obligatoria, es preciso introducir más mejoras para minimizar la carga administrativa. Todo método nuevo implica costes iniciales, por lo que la Comisión recomienda que las empresas que decidan aplicar voluntariamente la metodología lo hagan previa evaluación detallada del impacto sobre su competitividad y que, del mismo modo, los Estados miembros 
que la apliquen evalúen los costes y beneficios para las PYME" (Considerando 11).

La Recomendación parte de una expresión de una cierta desconfianza hacia el resultado conseguido al limitar su alcance a un documento de carácter voluntario, con un cierto aire a trabajo en construcción pendiente de mejoras y en el que "recomienda" a las empresas que evalúen previamente a su uso sistemático como afecta a su competitividad. Con parecida advertencia se acompañaban las Conclusiones del Consejo Europeo sobre el plan de acción para la economía circular (20.06.2026), donde destaca la necesidad de contar con "un marco de vigilancia para reforzar y evaluar los avances en la transición hacia una economía circular, minimizando al mismo tiempo la carga administrativa".

Por otra parte, impulsando esta metodología se busca unificar la pluralidad de indicadores existentes de comportamiento ambiental en un intento claro de dotarse de herramientas que unifiquen el mercado interior. Sin embargo, su carácter puramente voluntario parece actuar en sentido contrario al pretendido porque difícilmente va a jugar ese papel unificador una metodología que no compromete a los Estados Miembros, a las empresas y que no se incorpora con carácter obligatorio a ningún acto normativo. Se afirma, incluso, que "la presente Recomendación no se aplica a la ejecución de actos legislativos obligatorios de la UE que prevén una metodología específica para el cálculo del comportamiento ambiental de los productos en el ciclo de vida" (1.3).

El creciente peso que está teniendo la lucha contra las barreras no-tarifarias en los acuerdos impulsados por las organizaciones internacionales, sobre todo por la OMC ${ }^{1}$, que básicamente agrupan medidas administrativas y legales que se entiende que limitan o impiden los intercambios, se percibe también en la Recomendación de la huella ambiental de 2013 advirtiéndose que "la Comisión, consciente del mercado mundial, mantendrá informadas a las organizaciones internacionales acerca de esta iniciativa voluntaria". Se puede apreciar, por tanto, que al debate técnico que suscita la definición de indicadores también se superpone un debate político y jurídico sobre si esos indicadores pueden ser perjudiciales para la expansión globalizadora de unas reglas de mercado neoliberales que ponen bajo sospecha un amplio abanico de

\footnotetext{
${ }^{1}$ https://www.wto.org/english/thewto_e/whatis_e/tif_e/agrm9_e.htm
} 
medidas de intervención administrativa entre las que se contarían estos indicadores de comportamiento ambiental si su diseño no es adecuado. Por este motivo se ha defendido una metodología de la huella ambiental de ámbito internacional, con especificidades para adaptarse a las condiciones locales, que podría limitar la diversidad de enfoques reduciendo las barreras a los intercambios y facilitando el acceso a los mercados europeos (AGRAWALA, 2018).

La OCDE apunta a que la multiplicación de ecoetiquetas o esquemas de información dificulta que alguno de ellos alcance una cuota de mercado suficiente para convertirse en una exigencia de facto, pero que esa misma multiplicidad puede afectar a los costes y beneficios de los productores que se vean empujados a adoptarlos. También advierte que algunos instrumentos nuevos como la huella de carbono o la huella ambiental tienen potencialidades, pero presentan retos de compatibilidad y calidad de los datos (OCDE, 2016, 17).

En un estudio sobre las ecoetiquetas e instrumentos de información sobre el comportamiento ambiental, la OCDE indica que hay dos tendencias claras: la "intensificación", con un crecimiento sostenido de sellos y; la "extensificación", con la aparición de nuevas variedades, fundamentalmente ligadas a la comunicación cuantitativa sobre energía y carbono, basada en el ciclo de vida (OCDE, 2016, 6). E estudio muestra un rápido crecimiento del número de esquemas de comportamiento ambiental, especialmente desde finales de los años 90 y entre 2007 y 2010, seguido de un periodo de ralentización desde 2012. Los sistemas gestionados gubernamentalmente son una minoría. Ha habido un cambio de la gestión por organizaciones sin ánimo de lucro hacia sellos privados de empresas. También un cambio de instrumentos relacionados con la biodiversidad hacia los referidos al cambio climático, aunque en conjunto los que conciernen a los recursos naturales y el control de productos químicos siguen siendo mayoría. Muchos siguen sin basarse en análisis del ciclo de vida, lo que -se afirma- puede ser muy caro y "no necesariamente aportar grandes ganancias en transparencia ambiental".

La dificultad de que un esquema "one-size-fits-all" pueda adaptarse a diferentes entornos o producciones, junto con los elevados costes que implican ciertos indicadores y la proliferación de esquemas no compatibles, son factores que pueden levantar barreras en el mercado o limitar la competencia para pequeños productores, países emergentes, etc. También incidiría el hecho de que, de forma creciente, estos 
sistemas son propiedad de empresas privadas, con los riesgos añadidos que comporta de "green-washing" y competencia a la baja.

\section{APLICACIÓN DE LA METOdOLOGíA DE LA HUELLA AMBIENTAL: POSIBLES USOS Y ALCANCE}

La medición de la huella ambiental cobra interés para el Derecho si se utiliza para la articulación de políticas públicas o la adopción de decisiones administrativas. El aspecto jurídicamente más relevante realmente es el uso y alcance que adopte esa incorporación al ámbito público de una metodología que aporta datos de sostenibilidad.

Para ello el cálculo de la presión sobre la renovación del medio que produce una actividad, producto o territorio concreto debe conseguirse incorporando información de calidad. La inexistencia o dificultad de cuantificación de ciertos factores, como la biodiversidad, y los costes de conseguir información de calidad puede ser un elemento disuasorio para pequeños productores o países en desarrollo que puede exacerbarse en sistemas como el de la huella ambiental, que requieren un número mayor de cálculos para hacer el análisis del ciclo de vida. Por ello deberían buscar un equilibrio entre la exactitud, accesibilidad e imparcialidad (OCDE, 2016, 13). Las exigencias de transparencia e imparcialidad propios de los procedimientos administrativos de toma de decisión deberían replicarse cuando etiquetas, indicadores de comportamiento ambiental u otros instrumentos de similar naturaleza aspiran a jugar algún papel en la esfera pública. AGRAWALA (2018) se preguntaba si el carácter multi-dimensional de la información de la huella ambiental favorece elecciones de los consumidores más informadas o bien si puede provocar una sobrecarga de datos, también si sería preciso un sello o etiqueta que la distinga o cómo sería posible presentar la información subyacente que implica. Todas estas cuestiones nos remiten al entorno de incertidumbre en que se mueve todavía la huella ambiental en cuanto a su posible utilidad y alcance.

La Recomendación de la UE de 2013 sobre la huella ambiental de productos y organizaciones apunta un abanico de posibles ámbitos de aplicación (Anexo I). Algunos de ellos podríamos decir que son de naturaleza empresarial (ej. optimización 
de procesos a lo largo del ciclo de vida de un producto o de la cadena de suministro) o internos. "Las aplicaciones internas pueden incluir el apoyo a la gestión ambiental, la determinación de los puntos críticos desde el punto de vista ambiental, la mejora y el seguimiento del comportamiento ambiental, y pueden suponer, de manera implícita, oportunidades de ahorro" (Recomendación UE 9.4.2013, 12). Estas aplicaciones pueden ser útiles para realizar el seguimiento de sistemas como el EMAS en tanto que proporcionan información sobre la situación ambiental de la empresa.

En la vertiente externa, un número significativo de las aplicaciones posibles guardan relación con la aportación de información a los consumidores o a las administraciones. En el primer caso como herramienta de marketing, con efectos semejantes a los que tienen las etiquetas ecológicas, pero con la huella ambiental con un diseño más transversal o multi-dimensional (ej. documentación que acompaña al producto, páginas web y aplicaciones; regímenes de productos de prestigio que dan visibilidad a los productos que calculan su comportamiento ambiental en el ciclo de vida; informes anuales, informes de sostenibilidad, respuesta a cuestionarios de inversores o partes interesadas; información para sistemas de gestión ambiental o ecoetiquetas). En el segundo caso aportando datos en el seno de procedimientos administrativos o cumpliendo trámites de control de comportamiento ambiental (ej. alegaciones ambientales, en particular para garantizar la solidez e integridad suficientes; regímenes que exigen información sobre el comportamiento ambiental en el ciclo de vida).

Otro grupo de aplicaciones posibles es la configuración de incentivos basados en la mejora del comportamiento ambiental en el ciclo de vida. Finalmente, se señala también una posible aplicación, de interés para el impulso de la estrategia en construcción de economía circular, que sería usar la huella ambiental de productos para apoyar "un diseño de productos que minimice el impacto ambiental a lo largo del ciclo de vida". Tomando en consideración que el eco-diseño es uno de los pilares fundamentales de la economía circular, parece claro que una de las utilidades de la huella ambiental podría ser la comparación del impacto del diseño los productos, si bien la Recomendación de la UE $(2013,9)$ expresamente establece que "no pretende apoyar directamente las comparaciones o aseveraciones comparativas, es decir, alegaciones de la superioridad global o equivalencia del comportamiento ambiental de un producto respecto a otro". Se menciona también la contratación ecológica 
como uno de los campos en que se podría utilizar la metodología de la huella ambiental $(2013,12)$.

Las posibles aplicaciones indicadas de la huella ecológica parten de la base de que la UE ha descartado explícitamente usarla para la "ejecución de actos legislativos obligatorios de la UE que prevén una metodología específica para el cálculo del comportamiento ambiental de los productos en el ciclo de vida" y recomienda a los Estados miembros que la utilicen "en sus políticas voluntarias" (Recomendación 9.4.2013, 1.3, 3.1). Ese enfoque voluntario unido a las discusiones metodológicas mencionadas anteriormente puede limitar la funcionalidad de la huella ecológica que corre el riesgo de solaparse con otras iniciativas e indicadores más concretos o amparados en esquemas obligatorios que les proporcionan un mayor respaldo. En ese sentido, PRAG $(2013,22)$ apunta que estándares de resultado, como la huella de carbono o la huella ambiental, que no se centran en ningún aspecto concreto del proceso productivo pueden dar más flexibilidad a productores y proveedores para determinar cómo conseguir resultados, si bien eso podría tener repercusiones en un uso inconsistente de la metodología que permita enmascarar problemas a incumplidores ("greenwashing"), penalizando a cumplidores ("greenbashing"). Precisamente, los indicadores únicos reciben tres tipos de críticas (ECOTEC, 2001, 8): que no son necesarios ya que los usuarios pueden manejar más de una serie de datos; que para alcanzar una unidad de medida común deben sumar datos heterogéneos dando una visión distorsionada y que cada problema ambiental presenta sus singularidades por lo que agruparlos en un indicador único es inaducuado (ej. ningún aumento en la calidad del agua compensa la pérdida de capa de ozono).

La dificultad para realizar por el momento cálculos finos, ágiles y comparables limita la potencialidad de la huella ambiental como herramienta de visualización del comportamiento ambiental de productos. Incurre, de hecho, en heurísticos y simplificaciones, para calcular estimativamente la cantidad de hectáreas globales precisas para producir un producto o soportar actividades en un momento y espacio dado. Además, complica la visibilización de los puntos débiles más relevantes que determinan esa huella ambiental concreta. El avance de las tecnologías de la información y la mejora del tratamiento de datos puede tener un efecto positivo en el futuro facilitando alguno de los aspectos señalados. No obstante, probablemente su 
mayor virtualidad actual esté en la comparación de territorios u organizaciones reflejando tendencias de sostenibilidad que compelan a la acción global, que no en el regate corto empresarial más ligado al marketing. ECOTEC $(2001,39)$ apuntaba, en esa línea, que los indicadores ambientales pueden tener dos funcionalidades principales: comunicativa o de gestión empresarial; en el primer caso, deberían ser pocos y fáciles de comprender, mientras que en el segundo debería ser más desagregados (sectorial, regional...) y alineados con las exigencias de las políticas públicas. Se concluía que la huella ambiental encaja mejor en esa función comunicativa.

\section{REFLEXIONES CONCLUSIVAS: EL DERECHO ANTE LA DETERMINACIÓN DE LOS PARÁMETROS METODOLÓGICOS PARA LA MEDICIÓN Y EL USO DE LA HUELLA ECOLÓGICA}

La incorporación por el Derecho de la huella ambiental como indicador parte, como hemos descrito, de una situación de debate metodológico y discusión sobre su validez y alcance. En ese contexto de incertidumbre o cuestionamiento científico, su utilidad para la definición de políticas públicas y normas debe tender a superar una parte de las limitaciones señaladas. Como señala ESTEVE (2009, 108-109) habría que "retomar el rumbo decisorio propio del Derecho" que, sin negar la autoridad experta de las instancias científicas, use los métodos característicos del Derecho para adoptar decisiones con la legitimación propia de un sistema democrático. Este método "no pretende alcanzar la certeza, sino resolver los problemas jurídicos planteados" construyendo un entramado de ficciones sobre los que se aportan soluciones jurídicas (ESTEVE, 2010, 45). En ese método jurídico tienen especial relevancia las presunciones, procedimiento y forma de obtención de los datos que configuran la huella ecológica.

Un elemento, en concreto de la metodología de la huella ambiental impulsada por la UE, que favorece esa adopción de decisiones con el método propio del Derecho es la voluntad de hacer explícitos los "límites del sistema" (punto 4.3), esto es detallar "qué partes del ciclo de vida del producto están incluidas o excluidas del análisis" o estimar la incertidumbre (punto 7.4). Aunque la primera exigencia no es obligatoria, "se recomienda encarecidamente" consignarla. Unido a esto se establecen pautas 
para seleccionar y añadir "información ambiental adicional" que pueda ser relevante y quedar fuera de los modelos de evaluación de la huella ambiental basados en el ciclo de vida (punto 4.5). Esta información ambiental adicional deberá ser fundada y revisada con los requisitos de la norma ISO 14020 y el capítulo 5 de la norma ISO 14021:1999; ser específica, exacta y no engañosa y pertinente para la categoría de productos y; muy significativamente, comunicarse separadamente de los resultados basados en el ciclo de vida. Estas previsiones facilitan la verificación pública de la fiabilidad de los datos aportados y la comparabilidad de estos, lo que unido a las previsiones de calidad de los datos (punto 5.6), permite introducir mecanismos de validación y revisión.

Precisamente, la imparcialidad, veracidad, accesibilidad de esa información son una pre-condición para los posibles usos que se realizan de la huella ambiental. La Recomendación UE 2013 determina como principios de la huella ambiental de productos la pertinencia, integridad, coherencia, exactitud y transparencia, en clara sintonía con esto. El hecho de que la principal virtualidad de la huella ambiental, en tanto que indicador que condensa en un único parámetro la información subyacente, sea el comunicativo obliga a extremar esas garantías propias del Derecho en el procedimiento de incorporación de la información. Si bien en los últimos tiempos las tendencias ligadas a la "smart regulation" y los desarrollos de las tecnologías de la información facilitan la obtención de datos cada vez más robustos, lo cierto es que garantizar la neutralidad y solidez de la información que proporciona la huella ambiental requiere un reforzamiento de los procedimientos de toma de decisión públicos.

Por el momento las iniciativas públicas en relación con la huella ambiental (ej. Recomendación UE 2013) parten en su mayoría de un uso puramente voluntario sin trascendencia normativa. En la práctica, sin embargo, muchas de las iniciativas privadas nacen con un respaldo público también. Un proyecto francés sugería que podría ser interesante establecer una norma que obligue a las etiquetas ecológicas a realizar un análisis del ciclo de vida que cumpla determinados estándares (MEDDE, 2013), lo que podría ser un ámbito de expansión de la huella ambiental. Este es un ejemplo de por donde podría encaminarse la participación de los gobiernos con el objetivo de brindar consistencia en el mercado frente a la proliferación de marcas ambientales y, al mismo tiempo, limitar la distorsión del mercado que puede producir 
esta variedad, en línea con lo que pretende la Recomendación de la UE (PRAG, 2013,38).

De forma similar a lo que había sucedido con la eco-etiqueta y el EMAS, aunque con un nivel de apoyo institucional significativamente menor como denota el instrumento normativo elegido, la UE apuesta por dotar a la huella ambiental como indicador de comportamiento ambiental de un respaldo público explícito que le de solidez y comparabilidad. De hecho, la metodología incorpora aspectos que recuerdan por su concepción a los criterios ecológicos de las categorías de productos de la ecoetiqueta. Las reglas de categoría de la huella ambiental de los productos, con sus funciones de determinación de los límites, parámetros clave, etapas del ciclo de vida o uso de recursos y emisiones (punto 2), sigue patrones semejantes a los criterios de la eco-etiqueta, incluso en el nivel intermedio de detalle de las categorías de producto (punto 2.3). En este caso, se diseña una metodología para alcanzar la huella ecológica y se publica en una Recomendación, lanzando un proyecto piloto de aplicación, con la finalidad de refinar y expandir esta metodología. No obstante, se explicitan sus límites: no-obligatoriedad, no-fragmentación del mercado interior, alcance fundamentalmente comunicativo.

En línea con el reforzamiento de los métodos de decisión dotados de legitimación democrática propios del Derecho, la Recomendación de la UE $(2013,9-10)$ requiere apoyar cada requisito en "documentos de orientación y métodos similares de contabilidad ambiental que gozan de amplio reconocimiento", básicamente normas técnicas, lo que debería llevar a que los poderes públicos "supervisaran la participación de los sectores sociales afectados, así como la transparencia de tales procedimientos" de normalización para garantizar la consecución del interés general en esos ámbitos con el fin de que se produzca una "transformación, que no claudicación, de lo público" (TARRÉS, 2015, 153). Se trataría de transponer los valores públicos a los agentes privados por medio de la organización y el procedimiento, con garantías de neutralidad en la toma de decisiones (BARNÉS, 2015).

Uno de los aspectos jurídicamente relevantes en cuanto a la huella ecológica es el referido a sus efectos en las reglas de mercado y/o incremento de cargas administrativas. (PRAG, 2013,35) apunta que la dificultad de saber cómo se suplen las carencias informativas existentes puede afectar, en un contexto de proliferación 
de esquemas a nivel internacional, confundiendo a los productores y usuarios sobre si la huella ambiental es correcta o donde puede estar arrojando resultados desequilibrados.

En definitiva, parece que el apoyo público, en tanto la huella ambiental sea una metodología aún en construcción con una vocación de herramienta voluntaria de medición del comportamiento ambiental, debe sustanciarse en dar soporte fundamentalmente al perfeccionamiento de los mecanismos y procedimientos de garantía de la neutralidad, veracidad y comparabilidad de los datos que se utilizan. Un planteamiento éste que remite a debates doctrinales de más alcance como son los del papel de los poderes públicos en una época de reconfiguración de los límites entre el Estado y la sociedad, el del papel del Derecho ante la incertidumbre científica y el de la búsqueda de nuevas formas de luchar contra el deterioro ambiental y la sostenibilidad del planeta.

\section{BIBLIOGRAFIA}

AGRAWALA, S. (2018), "Environmental labelling and footprinting: Evolution and Policy Implications", Final Conference of the Environmental Footprint Pilot Phase, Brussels,

http://ec.europa.eu/environment/eussd/smgp/EFconference 2018.htm

ALONSO IBÁÑEZ, R. (2016), "Marco jurídico para el urbanismo social y participativo", en Gifreu, J., Bassols, M., Menéndez Rexach, Á. (dirs.), El derecho de la ciudad y el territorio. Estudios en homenaje a Manuel Ballbé Prunés, INAP-Fundación Democracia y Gobierno Local.

BARNÉS, J. (2015), "La transposición de valores públicos a los agentes privados por medio de elementos de organización y de procedimiento", en DARNACULLETA, M., ESTEVE PARDO, J., SPIECKER, I., Estrategias del Derecho ante la incertidumbre y la globalización, Marcial Pons.

CHAMBERS, N., LEWIS, K. (2001), Ecological Footprint analysis: towards a sustainability indicator for business, inédito, Best Foot Forward.

ECOTEC (2001), Ecological footprinting: A technical report to the STOA Panel (Draft), 
http://www.europarl.europa.eu/RegData/etudes/etudes/join/2001/297571/DG-4JOIN_ET(2001)297571_EN.pdf

ESTEVE PARDO, J. (2009), El desconcierto del Leviatán. Política y derecho ante las incertidumbres de la ciencia, Marcial Pons.

ESTEVE PARDO, J. (2015), "Decidir y regular en la incertidumbre. Respuestas y estrategias del Derecho Público", en DARNACULLETA, M., ESTEVE PARDO, J., SPIECKER, I., Estrategias del Derecho ante la incertidumbre y la globalización, Marcial Pons.

European Commission, Joint Research Centre, Institute for Environment and Sustainability (2010), ILCD Handbook: Analysing of existing Environmental Impact Assessment methodologies for use in Life Cycle Assessment, https://eplca.jrc.ec.europa.eu/uploads/ILCD-Handbook-LCIA-Background-analysisonline-12March2010.pdf

FOLKE, C., JANSSON, A., LARSSON, J., COSTANZO, R. (1997), "Ecosystem appropriation by cities", Ambio, v26/3, 1997

GALERA RODRIGO, S. (2016), "Políticas locales de clima: una razón (adicional) para renovar la planificación (¿local?) en España”, en Gifreu, J., Bassols, M., Menéndez Rexach, Á. (dirs.), El derecho de la ciudad y el territorio. Estudios en homenaje a Manuel Ballbé Prunés, INAP-Fundación Democracia y Gobierno Local.

GIAMPIETRO, M., SALTELLI, A. (2014), "Footprints to nowhere", Ecological Indicators, Volume $\quad 46, \quad$ November http://dx.doi.org/10.1016/j.ecolind.2014.01.030

GOLFINGER, WACKERNAGEL, GALLI, LAZARUS, LIN (2014) "Footprint facts and fallacies: A response to Giampietro and Saltelli (2014) "Footprints to Nowhere"', Ecological Indicators, Volume 46, November 2014, http://dx.doi.org/10.1016/j.ecolind.2014.04.025

GULLÓN MUÑOZ-REPISO, N. (2008), "¿Puede la tierra con nuestro modo de vida?. La huella ecológica", CRÍTICA, № 951, Enero-Febrero 2008

MEDDE (2013) "Affichage environnemental des produits de grande consommation Bilan au Parlement ", www.developpementdurable.gouv.fr/IMG/pdf/Affichage_environnemental.pdf 
OCDE (2016), Environmental labelling and information schemes. Policy perspectives, mayo 2016, https://www.oecd.org/env/policy-persectives-environmental-labellingand-information-schemes.pdf

PNUMA, GEO 6 (2019), Perspectivas del medio ambiente mundial GEO 6. Resumen para responsables de formular políticas, https://wedocs.unep.org/bitstream/handle/20.500.11822/27652/GEO6SPM SP.pdf?s equence $=6 \&$ is Allowed $=y$

PRAG, A., LYON, T., RUSSILLO, A. (2013), Multiplication of Environmental Labelling and Information Schemes (ELIS): Implications for Environment and Trade, OECD Environment Working Papers n. 106, https://dx.doi.org/10.1787/5jm0p33z27wf-en PRIEUR, M. (2018), Les indicateurs juridiques. Outils d'évaluation de l'effectivité du droit de l'environnement, Institut de la Francophonie pour le Développement Durable (IFDD).

RAWORTH K. (2012), A safe and just space for humanity: can we live within the doughnut?, Oxfam Discussion Papers, https://www-cdn.oxfam.org/s3fspublic/file attachments/dp-a-safe-and-just-space-for-humanity-130212-en 5.pdf

RAWORTH,K.(2017), Doughnut economics: seven ways to think like a 21st century economist, London, Penguin Random House.

TARRÉS VIVES, M. (2015), "El papel de la normalización internacional en el contexto de la seguridad y el comercio de productos", en DARNACULLETA, M., ESTEVE PARDO, J., SPIECKER, I., Estrategias del Derecho ante la incertidumbre y la globalización, Marcial Pons.

WACKERNAGEL, M.,REES,W.E. (1997), "Perceptual and structural barriers to investing in natural capital: economics from an ecological footprint perspective", Ecological Economics 20,3-24.

WWF (2000), Living Planet Report 2000, Gland, Switzerland. 